\title{
An Event-Driven Dual Coordination Mechanism for Demand Side Management of PHEVs
}

\author{
Klaas De Craemer, Student Member, IEEE, Stijn Vandael, Student Member, IEEE, Bert Claessens, \\ and Geert Deconinck, Senior Member, IEEE
}

\begin{abstract}
This paper addresses the challenges of integrating existing PHEV charging algorithms, which optimize PHEV charging per market timeslot (e.g. 15 minutes), into an environment with realistic communication conditions. To address this challenge, we propose a dual coordination mechanism, which controls a cluster of devices on two different operation levels: market operation and real-time operation. The market operation level uses an existing timeslot-based algorithm to calculate a charging schedule per timeslot. The real-time operation level translates this schedule into event-based control actions for a realistic communication environment, wherein a limited number of messages can be exchanged. A case study of 1000 PHEVs shows that it is possible to achieve results on par with the timeslot based algorithm but with significantly reduced communication with the PHEVs.
\end{abstract}

Index Terms-Event-Driven, Demand Response, Communication, Electric vehicles, Coordination

\section{INTRODUCTION}

B ECAUSE of growing concerns about the environment and the decreasing reserves of fossil fuel, the transport industry is shifting towards full or (plug-in) hybrid electric vehicles (PHEVs). At the same time, government policies and subsidies are expected to push the share of renewable electricity generation, such as wind and photovoltaic power, to about $18 \%$ in 2035 [1]. However, these energy sources are greatly affected by variability and limited predictability.

One enabling technology is Demand Side Management (DSM), wherein loads are shifted to periods of time where this is beneficial from a cost or grid stability perspective. DSM is already applied today, but typically only for highuse industrial consumers. (PH)EVs are well suited for this type of control due to their high energy requirements and relatively long charging times. A fleet of charging PHEVs managed by a DSM aggregator [2] could act as a single entity on the energy market(s), and react to fluctuations in generation and consumption. In current literature, several algorithms are proposed for this purpose. However, realistic communication conditions are typically neglected.

K. De Craemer and G. Deconinck are with the Department of Electrical Engineering ESAT-ELECTA, KU Leuven, Kasteelpark Arenberg 10, B-3001 Heverlee, Belgium (e-mail: klaas.decraemer@esat.kuleuven.be and geert.deconinck@esat.kuleuven.be

B. Claessens is with the Flemish Research Institute VITO (email:bert.claessens@vito.be).

S. Vandael is with the Department of Computer Science, KU Leuven, Celestijnenlaan 200A, B-3001 Heverlee, Belgium (e-mail: stijn.vandael@cs.kuleuven.be).

All researchers are with Energyville, Dennenstraat 7, 3600 Genk, Belgium.
In DSM of PHEVs, communication plays an important role; PHEVs send charging requirements to an aggregator, while aggregators need to communicate control signals to EVs in order to steer charging power. In terms of integrating algorithms into a realistic communication environment, we identified two challenges: continuous coordination, and a limited exchange of messages.

The first challenge is continuous coordination of PHEV charging. In energy markets, charging only needs to be optimized in terms of energy per hour. However, in a realistic communication environment, PHEVs arrive and depart continuously, and will send events at asynchronous times. Consequently, charging needs to be coordinated at two levels: a market level, per timeslot, and a real-time, event-driven level that is focused on responsiveness.

The second challenge is limiting the exchange of messages between PHEVs and aggregator. In a realistic communication environment, the underlying infrastructure places constraints on the communication, such as delays or maximum throughput. In the latter case, the exchange of messages should be limited by the coordination mechanism.

To cope with both challenges, we propose a dual coordination mechanism, which consists of a market operation level and a real-time operation level:

1) The market operation level entails actions with the goal of following beforehand traded volumes on the wholesale electricity markets, where trading takes place on relatively long-term scale (months, seasons) and amounts are expressed as energy quantities (usually $\mathrm{MWh}$ ) in timeslots of typically 1 hour or 15 minutes.

2) The real-time operation level entails the actions to comply with consumer preferences and respect local grid constraints. Because changes and control are relatively more instantaneous and dynamic at this level, real-time operation is usually expressed in terms of electrical power (e.g. $\mathrm{kW}$ ). Granularity is in the range of minutes to seconds. At this level, fast responses are important and the number of exchanged messages will be limited.

In summary, the contributions of this paper are:

- Description of an event driven dual coordination mechanism, which allows for market and real-time operation of the fleet. The market operation of the fleet is based on an existing algorithm.

- Evaluation of the mechanism in varying scenarios, with respect to the original algorithm. Results show a 3\% improvement in fleet charging costs. At the same time, 
the number of messages exchanged with the PHEVs was significantly reduced, by at least $64 \%$.

\section{RELATED WORK}

To control charging PHEVs in a smart grid, several methodologies have been proposed. This section gives a representative selection and positions our work in current research.

- In [3], DSM of (PH)EVs with the goal of minimizing losses in a distribution grid is proposed. The approach is centralized as all information is collected and processed by a single aggregator. The PHEVs are simulated alongside household consumption and compared to a scenario with uncoordinated charging. In [4], load factor and load variance are also taken into account.

- In [5], a system rooted in online market mechanism design is presented and applied to the coordinated charging problem, with a focus on incentivising users to truthfully report their demands. Probabilistic information on future arrivals is used to improve scheduling. The system is also compared to an offline benchmark that has prior knowledge on vehicle arrivals.

- In, [6] the authors look at a Vehicle-to-Grid (V2G) scenario that minimizes energy costs while reducing power losses. To this end, a highly dimensional allocation problem is tackled with approximate dynamic programming.

- In [7], a system is proposed based on congestion pricing and Quality of Service (QoS) concepts in TCP/IP networks. Each consumer determines his demand in the next time-slot with a user utility function. An implied central entity receives demand information from the appliances and sends back pricing or incentive signals. The principle used is dual decomposition.

- In [8], optimal demand-side management (DSM) is achieved using a model derived from game-theory. Each consumers scheduler is required to broadcast its consumption schedule to all other participants in the DSM programme. Schedule granularity is one hour.

- In [9], an EV charging algorithm based on queueing theory and statistical analysis is used to maximize network utilization. The use of message broadcasting would allow the scheme to be used over one-way low bandwidth communication links.

- The work of [10] looks at the effects of network performance on cost optimization in a smart grid, while [11] considers the cost of communication when updating energy price information of home appliances.

In all of the above work, algorithms are based upon the notion of timeslots, wherein time is divided into discrete intervals with a typical fixed length of one hour or sometimes 15 minutes. This division closely matches with aforementioned market operation. While there is some work concerning the link between communication and optimization in smart grids [9], [10], it does not apply to the short-term, realtime level of the coordination. However, in order to realise large-scale native field implementations there is a need to be able to coordinate devices (charging vehicles but also other appliances) on a continuous timescale.

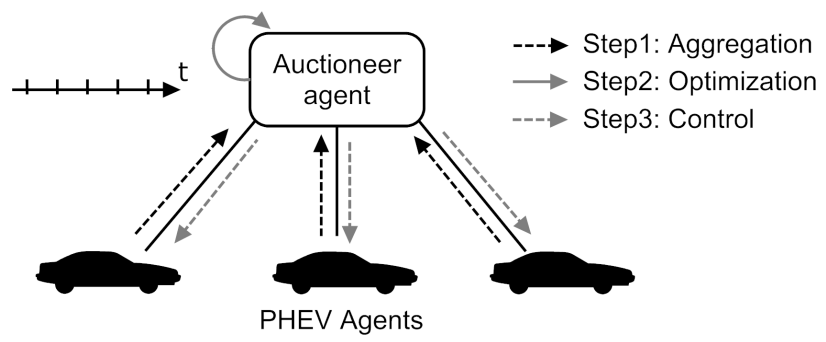

Fig. 1. Agent structure for the tree-step approach.

\section{BACKGROUND: THREE-STEP APPROACH}

In our previous work, a three-step charging algorithm was presented, which optimizes EV charging per market timeslot [12]. Based on this algorithm, we present a dual coordination mechanism in this paper (section IV). This section gives a brief overview of the three-step charging algorithm.

\section{A. Agent structure}

The agent structure is represented in Fig. 1. At the bottom of this structure are the PHEV agents, which represent the PHEVs at the local domestic level. At the top of this structure is the auctioneer agent, which represents the market operator who buys electricity for its PHEVs at an energy market. The goal of each type of agent is [12]:

- PHEV agent:

Charge the battery before departure time, while respecting local power limitations (e.g. maximum power of a household connection) and provide information on its flexibility to the auctioneer agent.

- Auctioneer agent: Charge PHEV fleet to minimize costs for energy supply (depending on production units managed by the energy supplier, prices at the wholesale electricity market ...) while respecting global constraints (e.g. power limits).

The charging algorithm consists of three steps (Fig. 1). In the first step, local PHEV constraints are aggregated towards the auctioneer agent. In the second step, the auctioneer agent uses the aggregated constraints to minimize energy supply costs. In the third step, a market-based incentive signal is propagated from the auctioneer agent towards the PHEV agents. The three steps are periodically repeated to cope with dynamics (e.g. arriving and departing PHEVs, changing electricity costs etc.).

\section{B. Step 1: PHEV constraints aggregation}

In step 1, the PHEV agents send their charging constraints to the auctioneer agent. These charging constraints consist of energy constraints and power constraints. Energy constraints represent the limits wherein charging energy can be shifted in time. Power constraints represent the limits within which a PHEV's charging power can be adjusted.

The individual energy constraints of each PHEV $i$ are expressed by the energy constraint vectors ${ }^{i} \mathbf{E}^{\max }$ and ${ }^{i} \mathbf{E}^{\min }$. These are visualized in Fig. 2a. The area in this graph contains all charging paths a PHEV can follow to achieve its required 


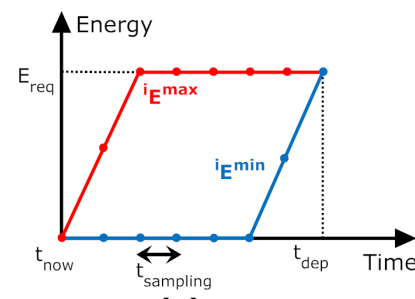

(a)

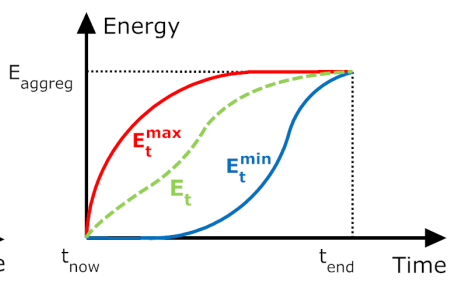

(b)
Fig. 2. A single energy constraints graph for an individual PHEV (a) and aggregated for an auctioneer agent (b). $E^{\min }$ and $E^{\max }$ are the energy limits while $E_{\mathrm{t}}$ is an optimal path satisfying the constraints for the whole fleet.

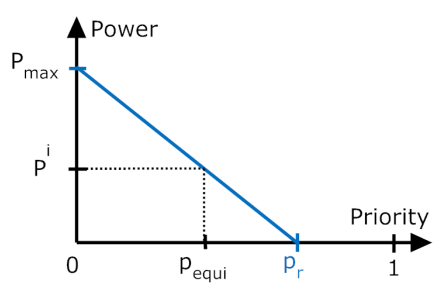

(a)

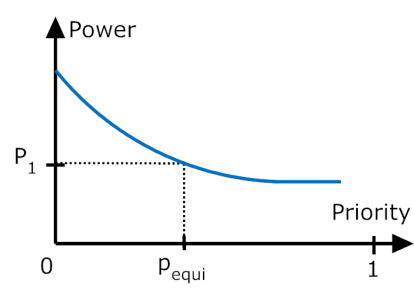

(b)
Fig. 3. Power constraints and corner priority $p_{r}$ for a single PHEV (a). The equilibrium priority $p_{\text {equi }}$ corresponds to charging power $P^{i}$. Aggregated power constraints, such as for the auctioneer agent, are shown in (b).

battery level $E_{\text {req }} ;{ }^{i} \mathbf{E}^{\text {max }}$ is the path of a PHEV $i$ if it were to start charging immediately at maximum power and then idle until departure $t_{\text {dep }}$, while ${ }^{i} \mathbf{E}^{\mathrm{min}}$ is for the case when charging is postponed as long as possible.

To represent the battery constraints of an entire PHEV fleet, the individual constraints are aggregated into collective battery constraints $\mathbf{E}^{\max }$ and $\mathbf{E}^{\mathrm{min}}$, shown in Fig. 2b:

$$
\mathbf{E}^{\max }=\sum_{i=1}^{n}{ }^{i} E^{\max }, \quad \mathbf{E}^{\min }=\sum_{i=1}^{n}{ }^{i} E^{\min }
$$

The power constraints of each PHEV $i$ are expressed by a demand vector ${ }^{i} \mathbf{P}^{\mathrm{dem}}$. This vector expresses a vehicle's desire to charge. For every possible priority $p$, an agent will determine its charging power, from low to high priorities. This is similar to a bid as used in Walrasian auctions.

One straightforward way of constructing the demand vector is by using a heuristic called the corner priority $p_{r}$ to determine the slope of a linear function, as depicted in Fig. 3a and equation (2):

$$
{ }^{i} f_{d}(p)= \begin{cases}P_{\max }-\frac{p P_{\max }}{p_{r}} & \text { if } 0 \leq p \leq p_{r}, \\ 0 & \text { if } p>p_{r}\end{cases}
$$

A higher $p_{r}$ value will give rise to a 'flatter' curve, indicating the willingness to consume at higher priorities. For convenience, $p$ is kept within $\left[0,1\left[\right.\right.$. Next, ${ }^{i} f_{d}$ is sampled at 100 points to obtain ${ }^{i} \mathbf{P}^{\mathrm{dem}}$, and interpolated when needed:

$$
{ }^{i} \mathbf{P}^{\mathrm{dem}}(p)=\left\{{ }^{i} f_{d}(p) \quad \forall p \in\{0,0.1,0.2, \ldots 0.99\}\right\}
$$

Different parameters can determine the willingness-to-pay of a PHEV and thus its demand vector, including:

- Maximum charge power $P_{\max }$, limited by the vehicles' power electronics or the local grid connection.
- Time until departure $\Delta t_{\text {dep }}$.

- Required energy $\Delta E$ needed by time $t_{\text {dep }}$.

In the work of [12], $p_{r}$ is directly combined from these parameters as in (4),

$$
p_{r}=\frac{\Delta E}{\Delta t_{\mathrm{dep}} P_{\max }}
$$

with $\Delta t_{\text {dep }}$ in hours, $P_{\max }$ in Watt and $\Delta E$ in Watt hour.

In this paper we are using the linear function of (5) to determine the corner priority $p_{r}$ and the agent demand curves.

$$
\begin{aligned}
& p_{r}=\frac{1}{2}-\frac{1}{2} \Delta t_{\mathrm{dep}}^{\prime}+\frac{1}{2} \Delta E^{\prime} \\
& \text { with } \Delta t_{\mathrm{dep}}^{\prime}=\left\{\begin{array}{cl}
\frac{\Delta t_{\mathrm{dep}}}{12} & \text { if } \Delta t_{\mathrm{dep}}<12 \mathrm{~h} \\
1 & \text { if } \Delta t_{\mathrm{dep}} \geq 12 \mathrm{~h}
\end{array}\right. \\
& \text { and } \Delta E^{\prime}=\frac{\Delta E}{E_{\max }}
\end{aligned}
$$

Using function (4) will emphasize charging close to $t_{d e p}$, as $p_{r}$ then behaves asymptotically when $t_{\text {dep }}$ approaches zero. Of course, different heuristics or a heterogeneous mix could be used as well. In case the device can only be switched on/off or consume at discrete rates, the demand vector can be a step or stair-shaped function.

As shown in previous work [13], the choice of demand function has an influence on the behaviour of the system, but this falls outside the scope of this work.

In case of insufficient time to get the needed charge, the demand vector is replaced by a flat curve at the maximum allowed power $P_{\max }$, meaning that the agent will charge no matter the priority. This emergency charging case is explained further in section V-A2.

\section{Step 2: Optimization of the PHEV fleet}

In step 2, the auctioneer agent determines a timeslot based charging plan for the entire PHEV fleet. This charging plan is based on a cost model $C$, which represents the costs for charging PHEVs. Examples of costs are distribution costs, generation costs and energy bought at energy markets (e.g. at the day-ahead market). The cost model and the aggregated energy constraints constitute the complete optimization problem:

$$
\min _{\mathbf{P}} C(\mathbf{P})
$$

subject to:

$$
\begin{array}{lr}
P_{t} \leq P_{t}^{\text {limit }} & \forall t \in\left\{1, \ldots, t_{\text {end }}\right\}, \\
E_{t}^{\min } \leq E_{t} \leq E_{t}^{\max } & \forall t \in\left\{1, \ldots, t_{\text {end }}\right\}, \\
E_{t+1}=E_{t}+P_{t} \Delta t & \forall t \in\left\{1, \ldots, t_{\text {end }}-1\right\} .
\end{array}
$$

where $t_{\text {end }}$ is the departure time of the PHEV which departs last, and $P_{t}^{\text {limit }}$ the power limits of the cluster. The latter can be built from the devices' energy constraints graph and aggregated into a cluster-wide $P_{t}^{\text {limit }}$. Dependent on the shape of the objective function $C$, different solution methods can be used to solve this problem (e.g. linear or quadratic programming). The result of solving this optimization problem is the control 
vector $\mathbf{P}_{\text {ctrl }}$, which defines control values from time $1\left(t_{\text {now }}\right)$ until $t_{\text {end }}$ for the entire PHEV fleet:

$$
\mathbf{P}_{\text {ctrl }}=\left\{P_{1}, P_{2}, P_{3} \ldots P_{t_{\text {end }}}\right\}
$$

\section{Step 3: Real-time control}

In step 3 , the auctioneer agent divides $P_{1}$ from (7) between the individual PHEVs of the fleet. For this purpose, the aggregated power constraints (Fig. 3b) are used to translate $P_{1}$ to an equilibrium priority $p_{\text {equi }}$.

Once the equilibrium priority is known, this priority value is sent as a control signal to all PHEV agents. Consecutively, each PHEV agent $i$ will locally match this priority in its personal demand vector ${ }^{i} \mathbf{P}^{\text {dem }}$ (Fig.3a, function $P$ in (8)) and start consuming at power $P^{i}$ :

$$
P^{i}=P\left({ }^{i} \mathbf{P}^{\mathrm{dem}}, p_{\text {equi }}\right)
$$

The result of these actions is a dispatch of $P_{1}$ between all PHEV agents.

The main advantage of this three-step approach is its scalibility: the computation of a charging plan for the EV fleet is independent of the total fleet size. Furthermore, the quality of solutions in terms of cost minimization are comparable to fully centralized solutions [12].

\section{REAL-TIME MARKET-BASED CONTROL}

As explained in section II, the above and existing algorithms make use of timeslots. Inherently, they do not consider changes that occur within a timeslot, such as arriving or departing vehicles or external events, which can be problematic in a direct field implementation. Decreasing the timeslot interval length to the order of seconds or minutes addresses this problem only partially, as it directly affects scalibility; agent communication and required computational power would rise quickly and prevent the use of large clusters of agents.

Therefore, to bring the three-step approach from section III to the real-time domain but retain its scalibility, we introduce the following additions:

1) Dual coordination by splitting the auctioneer agent into a real-time fleet manager and market operator agent.

2) Asynchronous updating of constraints.

3) Caching of constraints and equilibrium priorities.

4) Energy constraints graphs alignment and startpoint estimation.

In the next subsections these additions are discussed and then we compare the results with the timeslot based algorithm.

\section{A. Dual coordination}

The dual coordination approach separates the synchronously operating auctioneer agent of section III into a real-time part, the fleet manager, and a periodically moving part, the market operator. Additionally, to improve scalibility, concentrator agents are inserted. The resulting agent hierarchy is illustrated in Fig. 4.

The market operator is responsible for global optimization of the cluster. Because of its connection to the energy markets,

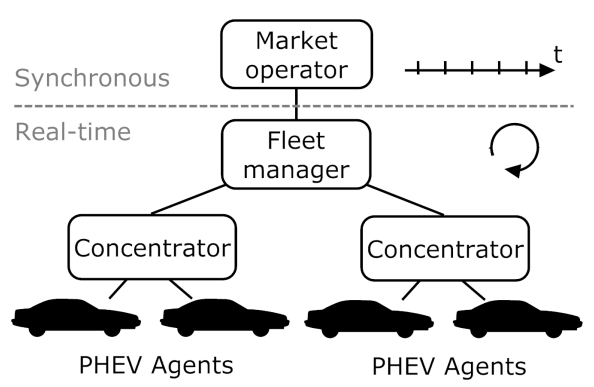

Fig. 4. Dual coordination agent structure for PHEV agents

the market operator will typically act on a periodic basis, for example every hour or 15 minutes. When it has determined a new strategy or optimum, it will pass a power or energy setpoint to the fleet manager.

The fleet manager is in the real-time domain and receives the result of the optimization by the market operator as a schedule containing power setpoints. Using the demand vector, it will attempt to steer the global consumption of the fleet in real-time towards this schedule by varying the equilibrium priority $p_{\text {equi }}$.

Concentrator agents aggregate the charging constraints from an underlying cluster of agents and appear as a single entity to the agent above it. They also perform the necessary agenthousekeeping by keeping track of arriving and departing PHEVs. By aggregating constraints data, concentrators improves the scalibility of the system. If necessary, multiple levels of concentrator agents could be used.

\section{B. Asynchronous updating}

All interaction in the real-time part of the agent structure is event-driven. Possible events are:

- PHEV arrival or departure: a vehicle is plugged in or out and the associated agent notifies the local concentrator.

- Modified constraints data: the agent (or the driver himself) changes the charging schedule and the demand vector ${ }^{i} \mathbf{P}^{\mathrm{dem}}$ is updated to reflect these changes.

- New equilibrium priority $p_{\text {equi: }}$ the fleet manager or concentrator agents distribute new priority data.

As opposed to a timeslot based control system, interaction between agents happens asynchronously. For demand vector data that passes from one agent to a higher level in the tree, this could mean that replies carrying the latest $p_{\text {equi }}$ are not necessarily related to the last submitted demand of that agent. Device agents would then apply this $p_{\text {equi }}$ on a demand vector that has not yet been incorporated in the fleet manager's optimization. Caching will further aggravate this effect. To keep demand vectors and priorities partially ordered, Lamport timestamps [14] are applied on their messages. By looking at the timestamps, agents can determine what previously submitted demand vector to use with a received $p_{\text {equi }}$ to determine $P^{i}$.

\section{Caching}

When an event occurs at the PHEVs, a constraints update is sent upwards, a new equilibrium determined and then 


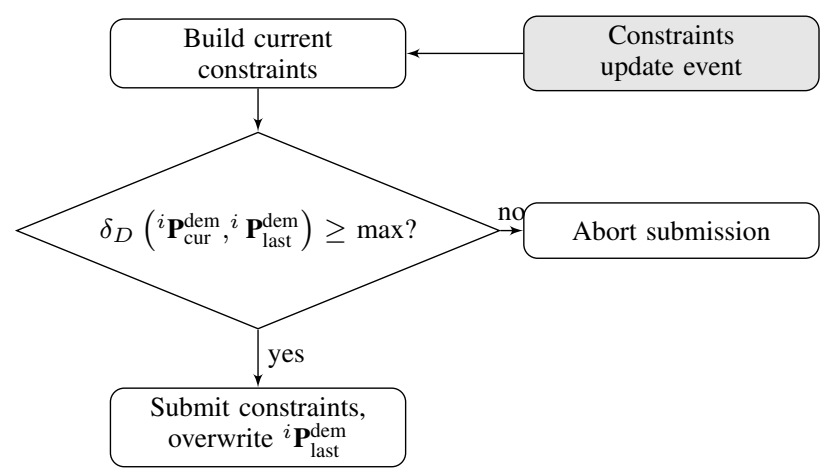

Fig. 5. Demand vector caching strategy for a real-time PHEV agent $i$

propagated downwards again. To avoid a continuous flood of these updates in case of large fleets, an intelligent caching scheme is implemented. Updates are sent when changes are deemed to have sufficient impact on either the demand vectors or the equilibrium priority.

1) Demand vectors: At the level of PHEV agents, the periodically rebuilt demand vector ${ }^{i} \mathbf{P}_{\text {cur }}^{\mathrm{dem}}$ is compared with the last one sent to the local concentrator, ${ }^{i} \mathbf{P}_{\text {last }}^{\mathrm{dem}}$. Their difference is quantified by determining the bounded discrete Fréchet distance between two demand vectors $\mathbf{P}_{1}^{\mathrm{dem}}(p)$ and $\mathbf{P}_{2}^{\mathrm{dem}}(p)$ as shown in (9).

$$
\begin{aligned}
& \delta_{D}\left(\mathbf{P}_{1}^{\mathrm{dem}}(p), \mathbf{P}_{2}^{\mathrm{dem}}(p)\right)= \\
& \min _{\substack{\alpha[0,1 \\
\beta[0,1[}}\left\{\max _{t \in[0,1[} d\left[\mathbf{P}_{1}^{\mathrm{dem}}(\alpha(t)), \mathbf{P}_{2}^{\mathrm{dem}}(\alpha(t))\right]\right\}
\end{aligned}
$$

with $d$ the Euclidian distance function and $\alpha(t), \beta(t)$ arbitrary discrete nondecreasing functions such that $\alpha(t), \beta(t) \in$ $\{0.1,0.2, \ldots 0.99\} \forall t \in\left[0,1\left[. \delta_{D}\right.\right.$ will then give the shortest coupling distance between the two demand vectors, which is a measure of how much $\mathbf{P}_{1}^{\mathrm{dem}}$ and $\mathbf{P}_{2}^{\mathrm{dem}}$ resemble each other. If $\alpha(t)=\beta(t)$, a simple maximum distance remains. The demand vector caching process is shown in Fig. 5.

Demand vector caching is also applied at the concentrator agents to decide if an aggregated vector should be sent upwards. Logically, the maximum allowed deviation should be larger than for an individual agent.

2) Equilibrium priorities: Upon receiving a new equilibrium priority from the fleet manager or another concentrator agent, a decision needs to be made whether to send this information immediately to agents lower in the tree or hold on to it for longer. During this process, illustrated in Fig. 6, impact numbers $\kappa$ are calculated according to (10). They are the difference between (estimated) power $P\left(\mathbf{P}^{\mathrm{dem}}, p\right)$ when the old equilibrium priority $p_{\text {old }}$ is kept and when the new one $p_{\text {new }}$ would be applied on agent $a_{i}$ 's last submitted demand vector ${ }^{i} \mathbf{P}_{\text {last }}^{\mathrm{dem}}$ instead.

$$
\kappa\left(a_{i}\right)=\left|P\left({ }^{i} \mathbf{P}_{\text {last }}^{\text {dem }}, p_{\text {old }}\right)-P\left({ }^{i} \mathbf{P}_{\text {last }}^{\text {dem }}, p_{\text {new }}\right)\right|
$$

For every newly received $p_{\text {equi }}$, a distinction is made between impact of the concentrator as a whole, $\sum \kappa\left(a_{i}\right)$, and individually per PHEV agent managed by that concentrator, $\kappa\left(a_{i}\right)$.

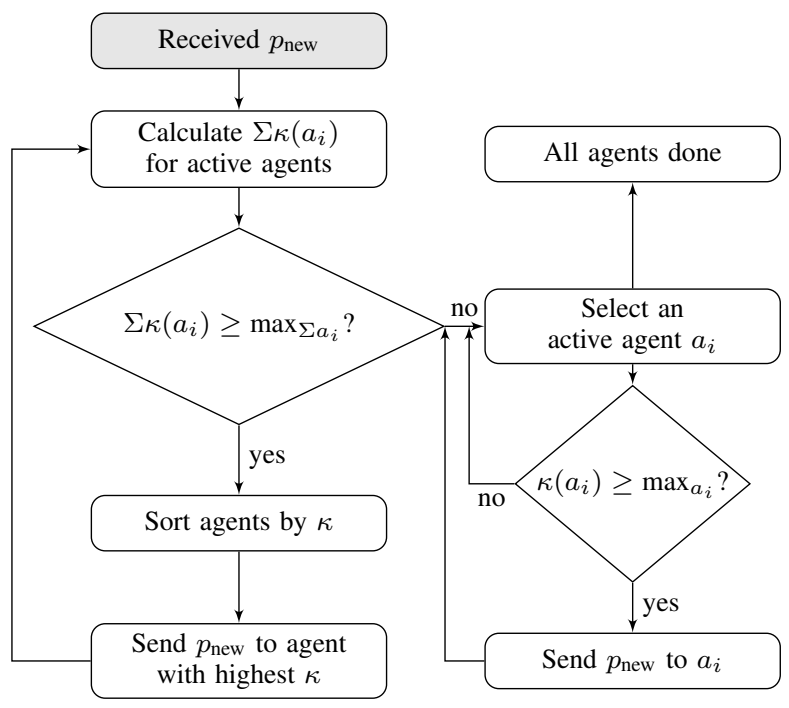

Fig. 6. Real-time concentrator agent equilibrium priority caching strategy

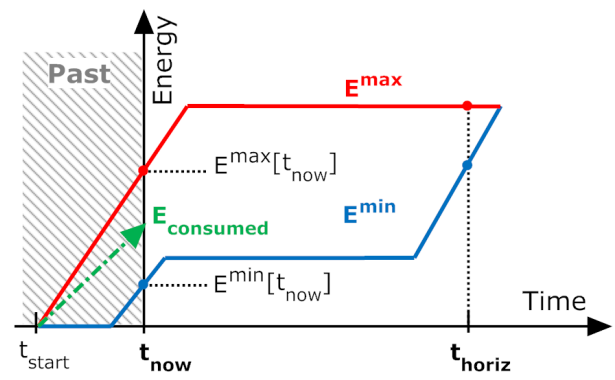

Fig. 7. Concentrator energy constraints estimation

\section{Energy constraints graphs}

The energy constraints introduced by [12] are tailored to the use of timeslots; the starting time $t_{\text {start }}$ is always the same for every agent that submits constraints. In the asynchronous situation, this does not hold; at the time of aggregation $t_{\text {now }}$, the energy constraints from the PHEVs stored at the concentrator differ in the time they were built (Fig. 7). To avoid contacting every device agent again when aggregating, some adjustments are made:

1) Sampling interval: If a 15 min. sampling interval would be used as in [12], the maximum error arising due the time discretization at a consumption of $3.3 \mathrm{~kW}$ amounts to $413 \mathrm{Wh}$ (as time is rounded to the nearest sampling interval multiple, but charge power is not allowed to exceed the charger's maximum of $3.3 \mathrm{~kW}$ ). By reducing it to 180 secs, the error is reduced to $83 \mathrm{Wh}$. The increase in amount of samples can be offset by parameterizing the graph and resample it upon aggregation, or by using non-uniform sample intervals.

2) Alignment \& energy estimation: During aggregation, the devices' individual energy constraints need to be re-aligned such that all of them start at the current time $t_{\text {now. }}$. As a consequence, the aggregated energy constraints graph can become "open-ended" on both sides (meaning that $E_{\min }$ and $E_{\max }$ do not coincide at the graph limits $t_{\text {now }}$ and $t_{\text {horiz }}$ ) as illustrated by Fig. 7 .

If the starting point of one or more devices' constraints 
curve lies in the past (e.g. $t_{\text {start }}$ ), it means that by $t_{\text {now }}$ the concentrator's devices will have accumulated some energy $E_{\text {consumed }}(11)$. Neglecting $E_{\text {consumed }}$ leads to an energy underestimation during optimizations by the market operator, in turn leading to overestimated power setpoints for the fleet operator.

As the concentrator holds the constraints and priorities associated with every agent $i$, it is possible to estimate $E_{\text {consumed }, i}$ by integrating $P\left({ }^{i} \mathbf{P}_{\text {last }, \mathrm{t}}^{\text {dem }}, p_{\text {last }, \mathrm{t}}\right)$, where ${ }^{i} \mathbf{P}_{\text {last }, \mathrm{t}}^{\text {dem }}$ is the last submitted demand vector at time $t$ of agent $i$ for which a corresponding priority $p_{\text {last,t }}$ was sent back. This is shown in equation (12). Deviations can occur when there is a delay between the concentrator sending priorities and the devices acting upon it.

$$
\begin{array}{r}
\mathbf{E}^{\max }\left[t_{\text {now }}\right] \geq E_{\text {consumed }} \geq \mathbf{E}^{\min }\left[t_{\text {now }}\right] \\
\text { and } E_{\text {consumed }}=\sum_{i} E_{\text {consumed }, i} \\
E_{\text {consumed }, i}=\sum_{t=t_{\text {start }}}^{t_{\text {now }}} P\left({ }^{i} \mathbf{P}_{\text {last }, \mathrm{t}}^{\text {dem }}, p_{\text {last }, \mathrm{t}}\right) \Delta t
\end{array}
$$

Eventually the aggregated constraints curve at $t_{\text {now }}$ can be corrected by adding $\sum_{i}\left(E_{\text {consumed }, i}-{ }^{i} \mathbf{E}^{\min }\left[t_{\text {now }}\right]\right)$ to ${ }^{i} \mathbf{E}^{\min }\left[t_{\text {now }}\right]$ of the aggregated curve, and restoring monotonicity if necessary.

\section{Evaluation}

The real-time market-based coordination system explained in sections III and IV has been implemented in a combined MATLAB/Java-based simulation. All event-driven agent logic is written in Java, while the market-level optimisation algorithms are implemented in MATLAB.

\section{A. Scenario and objectives}

Before we discuss the results, the scenario settings \& objectives for the market operator and the PHEV agents are explained.

1) Market operator: As depicted in Fig. 2b, the market operator defines a global power objective by esentially determining a valid optimal path $E_{t}$ within the aggregated energy constraints graph and is free to use any objective for this. Because energy markets are typically based on timeslots during which prices apply, it makes sense for the objective function to do as well.

When minimizing charging costs according to a Time-OfUse scheme is the sole business case, a purely linear costmodel is sufficient, in which case the market operator solves an LP problem of the form:

$$
\min _{\mathbf{P}, \mathbf{E}}\left(\sum_{t=0}^{t_{\text {horiz }}} m_{t} E_{t}+\sum_{t=1}^{t_{\text {horiz }}} \alpha\left|P_{t-1}-P_{t}\right|\right)
$$

with $E_{t}$ the energy consumed by the fleet, $m_{t}$ the energy price during time slot $t$ and using the constraints as in (6). The power limitation vector $P^{\text {limit }}$ is derived from aggregated information of the individual agents and/or from overloadavoiding constraints that apply (e.g. transformer or parking grid connection in case all vehicles are physically in the same

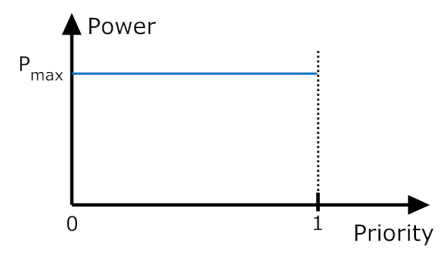

(a)

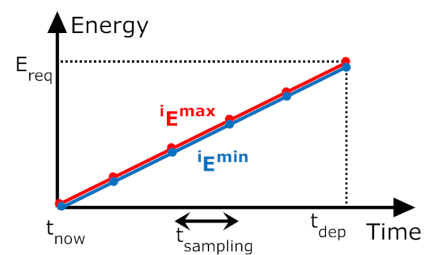

(b)
Fig. 8. PHEV emergency charging demand vector (a) and energy constraints graph (b).

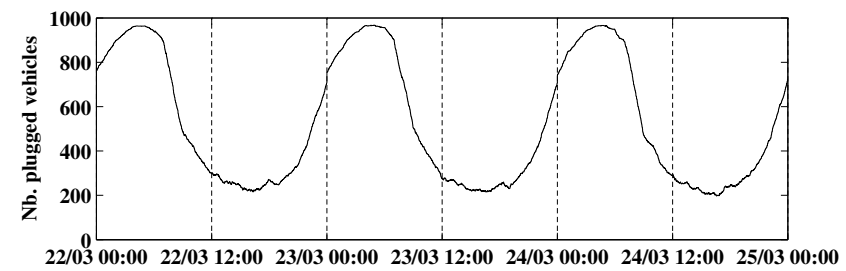

Fig. 9. Number of plugged-in and chargeable vehicles during simulation.

part of the grid). The regularisation term $\alpha\left|P_{t+1}-P_{t}\right|$ favors smooth solutions.

Other valid market operator goals include the minimisation of the variance between a portfolio of renewable energy sources (wind, solar...) and a number of fixed and controllable devices (households, PHEVs...) or limiting peak load. Stochastic information or learning algorithms could be incorporated as well, but the optimization aspect itself is outside the scope of this work.

2) PHEV device agents: PHEVs have individual objectives: charging in time and/or at low cost by providing bid data. The maximum charging power of a PHEV is chosen at $3.3 \mathrm{~kW}$, corresponding to a connection through a regular $230 \mathrm{~V}$ wall socket and the usable battery content equals $12 \mathrm{kWh}$.

The vehicles' driving behaviour is simulated using realistic profiles [15], in which measured driving data was used to build synthetic availability profiles. Battery state upon arrival is calculated assuming an average trip speed of $42 \mathrm{~km} / \mathrm{h}$ and driving efficiency of $4 \mathrm{~km} / \mathrm{kWh}$ (charging energy). Drivers plug in their cars at home (making most of the vehicles available between 19h-5h, Fig. 9) and give information on their next departure time $t_{\text {dep. }}$. A desired charge level is not supplied so charge-to-full is assumed. As home-charging is limited to $3.3 \mathrm{~kW}$ this might not always be feasible. In such a case, when there is not or only just enough time to obtain a full charge, the vehicle agent switches to emergency charging mode, independent from the fleet manager. The ${ }^{i} \mathbf{E}^{\min }$ and ${ }^{i} \mathbf{E}^{\text {max }}$ curves overlap and from then on charging proceeds at the maximum allowed power $P_{\max }$. Obviously, such a vehicle has no flexibility and can/will not react to $p_{\text {equi }}$ anymore. Fig. 8 illustrates this case.

The simulations were also performed using PHEV parameters of $6.6 \mathrm{~kW}$ power/20kWh battery, $2 \mathrm{~kW} / 12 \mathrm{kWh}$ and a heterogeneous mix of the three types, but apart from a different power profile the same conclusions were obtained. 


\section{B. Results}

The goal of the evaluations is to show that the original algorithm from [12] can be applied in a continuous realistic setting with the additions from section IV. A comparison with a timeslot based implementation is made to verify the performance.

1) General behaviour: A first setting consists of 1,000 PHEVs, distributed over 4 concentrator agents. The optimization at the market operator (13) employs day-ahead energy price information from the BELPEX [16], using a 24-hour horizon. Fig. 10 shows the power profiles and equilibrium priorities for both the $15 \mathrm{~min}$. timeslot based implementation and its real-time counterpart. The simulation starts on the 21th of March and runs for 4 consecutive days. Day one is left out due to transient effects of empty batteries at the start of the simulation. A detailed view is shown in Fig. 11. On the graph for the timeslot based system it is visible that the equilibrium priority and measured power consumption of the charging PHEVs vary at discrete times. The measured power however always deviates downwards from the market operator setpoint during the timeslots because vehicles achieve a full battery and/or leave within the length of a timeslot. This is expected as there is no way to react within the timeslots themselves. Similarily, vehicles arriving within a timeslot have to wait.

The real-time implementation uses the same discretized optimization in the market operator but the fleet manager adjusts $p_{\text {equi }}$ during the timeslots as it reacts on incoming messages. Therefore abovementioned deviations are avoided provided there is still flexibility in the cluster, i.e. when $0 \%<$ $p_{\text {equi }}<99 \%$. If this is not the case, the deviation is still known to the fleet manager, which is visible as the difference between 'Fleet manager setpoint' and 'Market operator setpoint' in Fig. 10.

Another effect occurs in the real-time implementation: because of the caching from section IV-C, $p_{\text {equi }}$ differs slightly throughout the hierarchical agent structure. The decision to hold newer $p_{\text {equi }}$ from certain devices is made by the concentrator agents, leading to a discrepancy between what the fleet manager believes is consumed by the devices and the real measured power. The amount of messages (through the aggressiveness of the caching mechanism) can therefore be traded against desired accuracy of the fleet manager. We previously showed this possibility in [13].

2) Comparison: In this part of the evaluation, the real time implementation is compared to the timeslots version regarding the quality of the solution. First, total charging costs are assessed by repeating the simulation setting from section V-B1 with a randomized set of 1,000 driving profiles (out of a set of 250,000 synthetic profiles [15]), and for 4 random consecutive days of the year. For the last 3 days of the simulation, the amount of device agent messages and the total charging cost is taken. Fig. 12 shows the distribution of these metrics for the simulations, and Fig. 13 shows the cost difference between the two approaches on a per simulation basis.

Table I shows a summary of the metrics for the whole set of randomized simulations. Despite the caching mechanism non-idealities, the charging costs have been reduced slightly
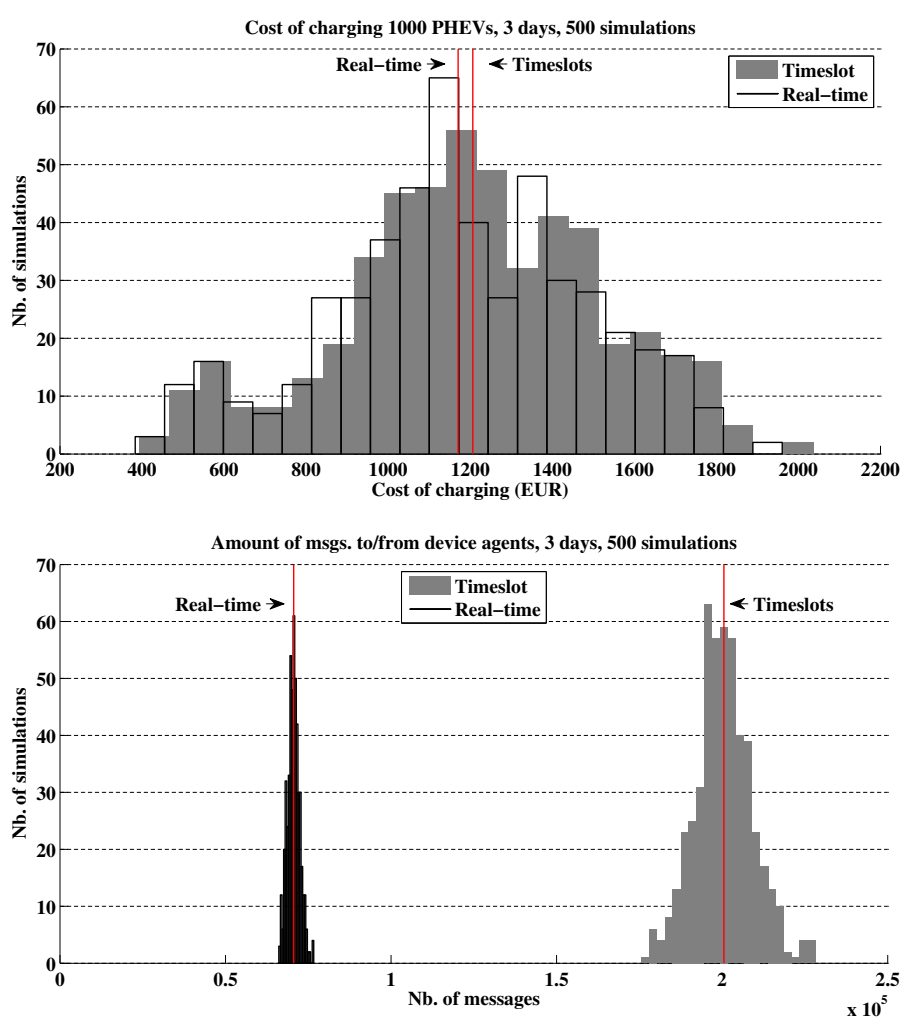

Fig. 12. Cost of charging (top) and messages sent and received (bottom) by 1,000 PHEVs over 3 random consecutive days, 500 simulations. The mean values are marked with a vertical line.

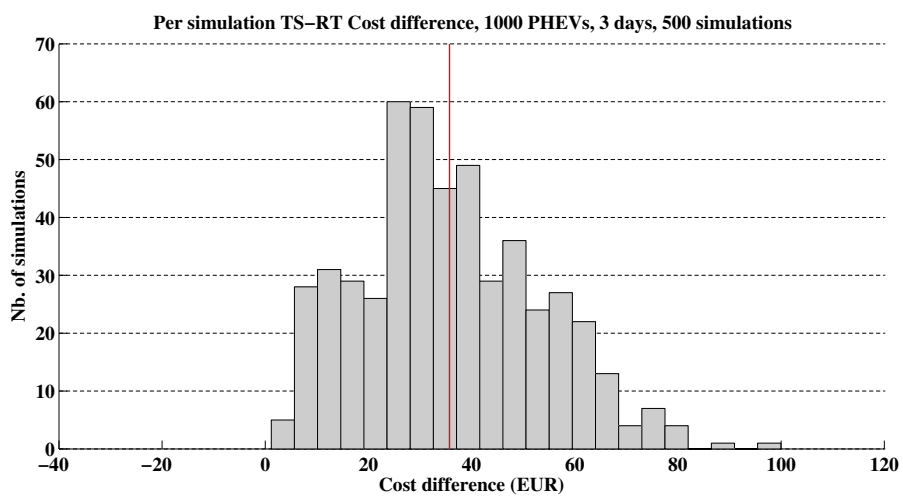

Fig. 13. Cost difference between the timeslot and real-time implementation on a per simulation basis $(1,000$ PHEVs, 3 random consecutive days, 500 simulations).

(by 3\%) with respect to the timeslot implementation. Simultaneously, the amount of messages exchanged with device agents was about $65 \%$ less. At the bottom of the previous figure, Fig. 10, a communication load graph has been included to show the distribution of messages during simulation. It is visible that the messages in case of the real-time coordination are centered around the times where energy prices are low. This makes sense as this is where the fleet manager sends new priorities to the cluster, illustrating that messaging now mainly occurs when it will have an impact.

In case of a communication failure at or with one of the concentrator agents, underlying PHEVs will continue charging using the last equilibrium priority data known to them. Device 

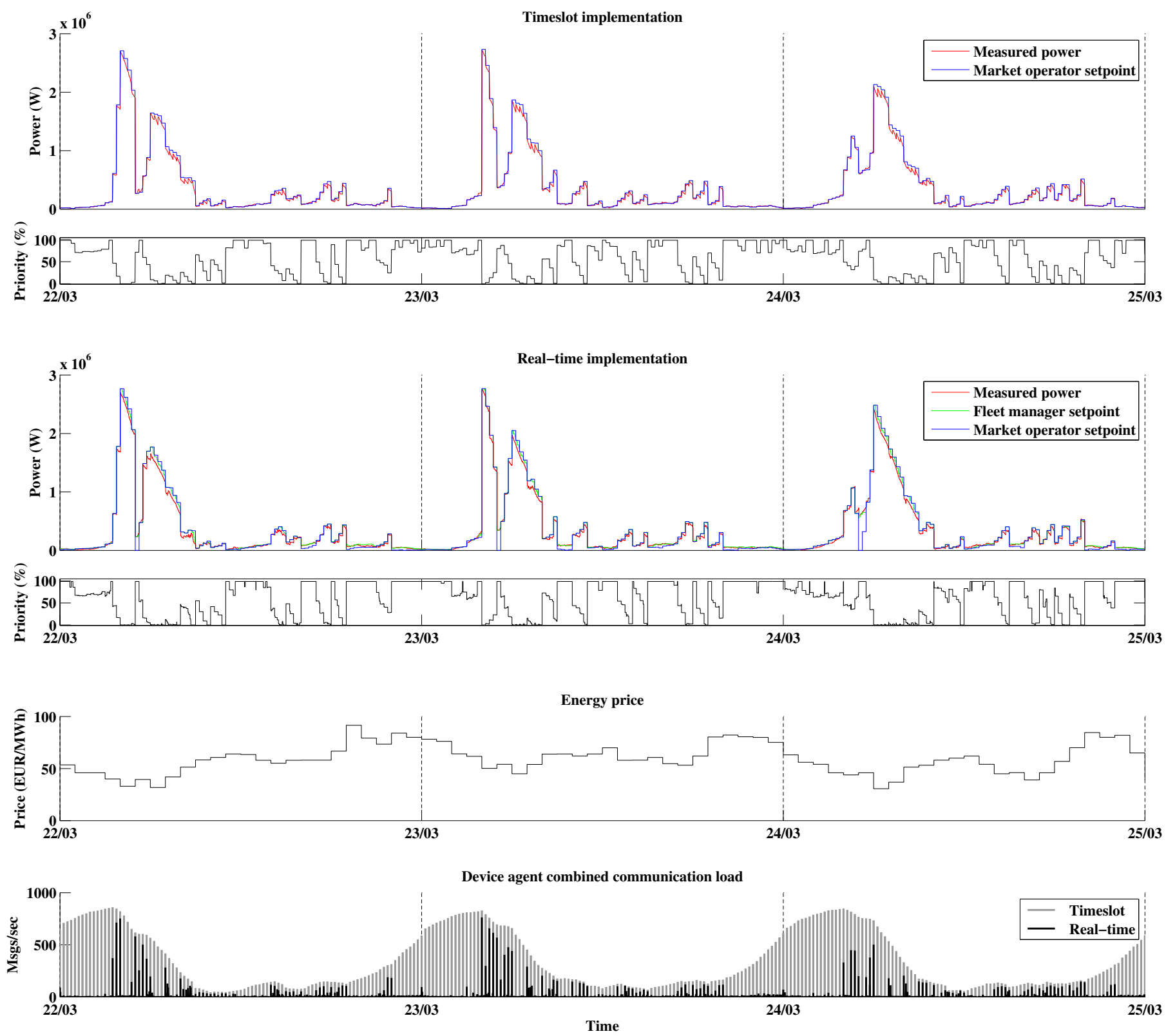

Fig. 10. Power consumption and equilibrium priority during 3 simulated days for a real-time and timeslot implementation, and the communication load on the device agents.
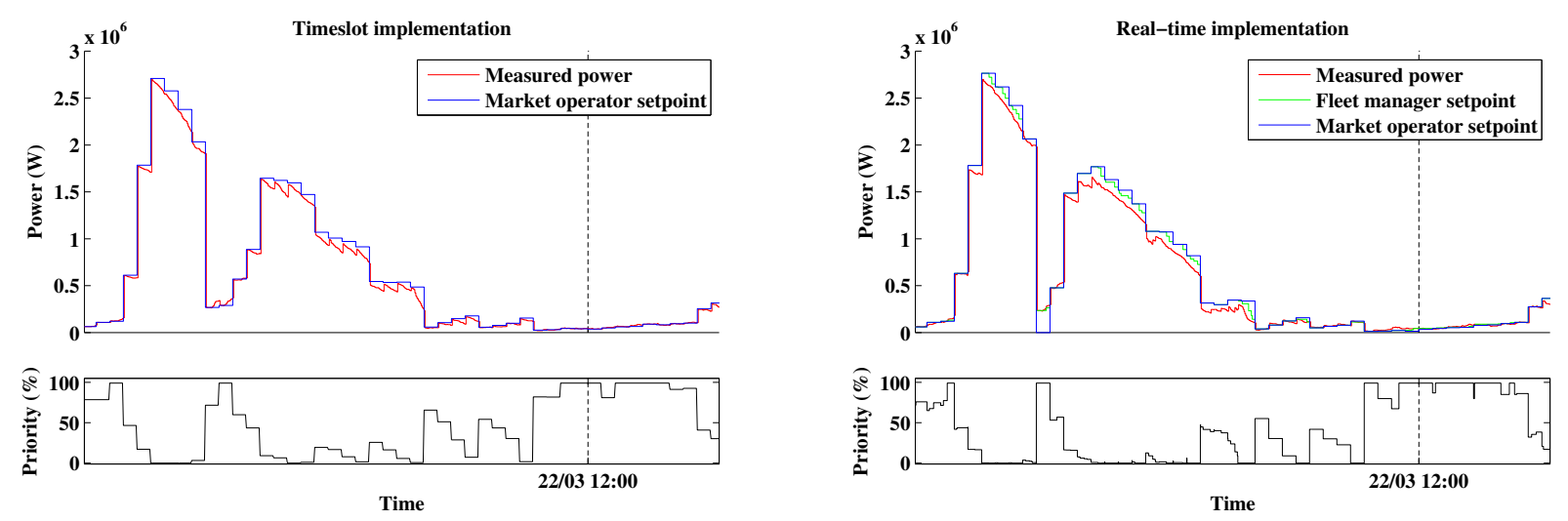

Fig. 11. Detailed views of the power profiles of Fig. 10 
TABLE I

RANDOMIZED SCENARIOS RESULTS

\begin{tabular}{lrrr} 
Averaged metric & Timeslots & Real-time & Improvement \\
\hline Charging cost & $€ 1,206.3$ & $€ 1,170.6$ & $2.96 \%$ \\
Nb. device messages RX & $96,956.0$ & $33,237.5$ & $65.72 \%$ \\
Nb. device messages TX & $103,582.7$ & $37,352.3$ & $63.94 \%$ \\
\hline
\end{tabular}

agents will still activate emergency charging when needed.

\section{Applicability to other scenarios}

The dual coordination approach's responsiveness allows to quickly react on external changes and thus offer some specific quick-response services such as shedding or increasing load. For example in the Netherlands, the imbalance prices are updated every minute, but the imbalance cost consolidation is done every 15 minutes [17]. Therefore the fleet manager can steer the power consumption of the cluster so that the quarterly imbalance cost is as low as possible. A retailer or Balancing Responsible Party (BRP) is able do the same within its portfolio.

\section{CONCLUSIONS \& FUTURE WORK}

In this paper, we presented a coordination mechanism which controls EVs on two different operation levels. The first level, "market operation", optimizes EV charging for discrete market time slots. Herein, we use an existing three-step algorithm. The second level, "real-time operation", translates this schedule into event-based control actions. In the latter, caching techniques are used to reduce the number of messages. This approach produces less overhead and results in similar or better performance than a comparable timeslot-only approach.

All results so far have focused on the use of $(\mathrm{PH}) \mathrm{EVs}$ as devices under control, having a relatively high degree of flexibility at the household DSM level. It is however also possible to apply the three-step approach to other types of consumers or generators [18]. As long as the flexibility of the underlying process can be translated into the power and energy constraints from section III, they can be integrated in the control system. Knowledge about the latter process or the intricacies of the demand vector function stays local and is therefore hidden from the upper control levels, allowing a 'standardized' constraints interface to be used. The energy constraints graph estimation from section IV-D2 will likely need to be adapted though.

The dual coordination approach can be expanded further; for example, the fleet manager could contain a feed-back mechanism to compensate for the differences that occur due to the caching. This would require measurements about actual consumption by the PHEVs.

Future \& ongoing work focuses on situations during which the state of the local distribution grid causes additional complications. For example, to protect the grid, the PHEV charger can implement droop control, capping charge power when the voltage sags too much. During these times, constraints at the real-time level take priority over the market level operation. Because of the event-driven approach, the updated demand vectors (PHEV or aggregated) can be quickly responded to by the fleet manager and an updated equilibrium priority will ensure that other agents make up for the loss in planned consumption. Similarily, in case multiple competing aggregators each control a number PHEVs within the same distribution grid, quick interaction between distribution grid dynamics (transformer limits, voltage profiles, droop control ...) and the market operator levels of both aggregators would be beneficial.

\section{REFERENCES}

[1] IEA, The IEA World Energy Outlook 2011., 2011.

[2] R. Bessa and M. Matos, "The role of an aggregator agent for EV in the electricity market," in 7th Mediterranean Conference and Exhibition on Power Generation, Transmission, Distribution and Energy Conversion (MedPower 2010). IET, 2010, pp. 126-126.

[3] K. Clement, E. Haesen, and J. Driesen, "Coordinated charging of multiple plug-in hybrid electric vehicles in residential distribution grids," in 2009 IEEE/PES Power Systems Conference and Exposition. IEEE, Mar. 2009, pp. 1-7.

[4] E. Sortomme, M. M. Hindi, S. D. J. MacPherson, and S. S. Venkata, "Coordinated Charging of Plug-In Hybrid Electric Vehicles to Minimize Distribution System Losses," IEEE Transactions on Smart Grid, vol. 2, no. 1, pp. 198-205, Mar. 2011.

[5] S. Stein, E. Gerding, V. Robu, and N. R. Jennings, "A model-based online mechanism with pre-commitment and its application to electric vehicle charging," in Proceedings of the 11th International Conference on Autonomous Agents and Multiagent Systems - Volume 2. International Foundation for Autonomous Agents and Multiagent Systems, 2012, pp. 669-676.

[6] V. W. Wong, "An approximate dynamic programming approach for coordinated charging control at vehicle-to-grid aggregator," in 2011 IEEE International Conference on Smart Grid Communications (SmartGridComm). IEEE, Oct. 2011, pp. 279-284.

[7] Z. Fan, "A Distributed Demand Response Algorithm and Its Application to PHEV Charging in Smart Grids," IEEE Transactions on Smart Grid, vol. PP, no. 99, pp. 1-11, 2012.

[8] A.-H. Mohsenian-Rad, V. W. S. Wong, J. Jatskevich, R. Schober, and A. Leon-Garcia, "Autonomous Demand-Side Management Based on Game-Theoretic Energy Consumption Scheduling for the Future Smart Grid," IEEE Transactions on Smart Grid, vol. 1, no. 3, pp. 320-331, Dec. 2010.

[9] K. Turitsyn, N. Sinitsyn, S. Backhaus, and M. Chertkov, "Robust Broadcast-Communication Control of Electric Vehicle Charging," in 2010 First IEEE International Conference on Smart Grid Communications. IEEE, Oct. 2010, pp. 203-207.

[10] D. Niyato, P. Wang, Z. Han, and E. Hossain, "Impact of packet loss on power demand estimation and power supply cost in smart grid," in 2011 IEEE Wireless Communications and Networking Conference. IEEE, Mar. 2011, pp. 2024-2029.

[11] H. Li and R. C. Qiu, "Need-Based Communication for Smart Grid: When to Inquire Power Price?" in 2010 IEEE Global Telecommunications Conference GLOBECOM 2010. IEEE, Dec. 2010, pp. 1-5.

[12] S. Vandael, B. Claessens, M. Hommelberg, T. Holvoet, and G. Deconinck, "A Scalable Three-Step Approach for Demand Side Management of Plug-in Hybrid Vehicles," IEEE Transactions on Smart Grid, pp. 1-9, 2012.

[13] K. De Craemer and G. Deconinck, "Balancing Trade-offs in Coordinated PHEV Charging with Continuous Market-based Control," in Proceedings of the Third IEEE PES Innovative Smart Grid Technologies (ISGT) Europe Conference, 2012.

[14] L. Lamport, "Time, clocks, and the ordering of events in a distributed system," Communications of the ACM, vol. 21, no. 7, pp. 558-565, Jul. 1978.

[15] J. Van Roy, N. Leemput, S. De Breucker, F. Geth, T. Peter, and J. Driesen, "An Availability Analysis and Energy Consumption Model for a Flemish Fleet of Electric Vehicles," in European Electric Vehicle Congress (EEVC), Brussels, 2011, p. 12.

[16] BELPEX, "Belgian Power Exchange," 2013. [Online]. Available: www.belpex.be

[17] TenneT, "Productinformatie regelvermogen," p. 7, 2011. [Online]. Available: http://www.tennet.org/tennet/publicaties/technische $\backslash \_p u b l i c a t i e s /$ overige $\backslash$ ppublicaties/productinformatie $\backslash$ _regelvermogen.aspx 
[18] B. Claessens, S. Vandael, F. Ruelens, and M. Hommelberg, "Selflearning Demand Side Management for a Heterogeneous Cluster of Devices with Binary Control Actions," in IEEE PES Innovative Smart Grid Technologies (ISGT 2012), 2012.

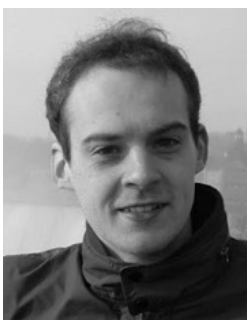

Klaas De Craemer Klaas De Craemer received the M.Sc. degree in Electrical Engineering from the KU Leuven, Belgium. In 2009 he started working towards a PhD degree at the department of electrical engineering (KU Leuven). His research interests include architectures and control systems for demand participation of smart homes and electrical vehicles, including their impact on grid stability.

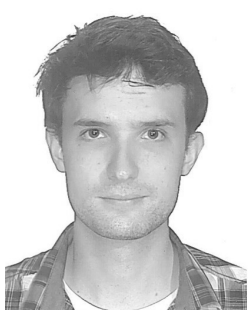

Stijn Vandael Stijn Vandael originates from Zonhoven, Belgium. He received the Master degree in industrial engineering from the Katholieke Hogeschool Limburg (KHLim), and the M.Sc. degree from the KU Leuven. Currently, he is working towards a PhD degree at the department of Computer Science (KU Leuven) in close cooperation with the department of Electrical Engineering (KU Leuven). His research interests include coordination in multiagent systems, plug-in hybrid electric vehicles and smart grids.

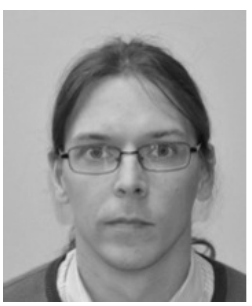

Bert Claessens Bert Claessens originates from Neeroeteren, Belgium. He obtained his MSc. and $\mathrm{PhD}$. in applied physics from the University of Technology of Eindhoven, The Netherlands, in 2002 and 2006, respectively. In 2006 he started working at ASML Veldhoven, The Netherlands, as a design engineer. Since June 2010 he has been working as a researcher at the Vlaamse Instelling voor Technologisch Onderzoek (VITO). His research interests include algorithm development and data analysis.

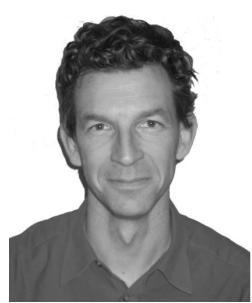

Geert Deconincks Prof.dr.ir. Geert Deconinck is full professor (gewoon hoogleraar) at KU Leuven (Belgium). As staff member of ESAT/ELECTA (Electrical Energy and Computing Architectures), he performs research on designing dependable system architectures for industrial automation and control, assessing their dependability attributes and characterizing infrastructure interdependencies. He received his M.Sc. in Electrical Engineering (1991) and his $\mathrm{Ph} . \mathrm{D}$. in Engineering (1996) from KU Leuven, and was postdoctoral fellow of the Fund for Scientific Research - Flanders (1997-2003). He is chairman of the TI society BIRA on industrial automation and a member of the IEEE SMC TC on Infrastructure Systems and Services, fellow of the Royal Flemish Engineering Society, the Institute of Engineering and Technology (IET), and a senior member of the IEEE (Reliability, Computer and Power \& Energy Societies). 